Gut, 1979, 20, 833-839

\title{
Suppressive effect of alcoholic liver disease sera on lymphocyte transformation ${ }^{1}$
}

\author{
G. P. YOUNG, ${ }^{2}$ F. J. DUDLEY, ${ }^{3}$ AND M. B. VAN DER WEYDEN
}

From Monash University Department of Medicine, Alfred Hospital, Victoria, Australia

SUMMARY The effect of alcoholic patient sera on in vitro lymphocyte transformation was studied using mitogen-induced uptake of ${ }^{3} \mathrm{H}$-thymidine to measure blastogenesis. With pokeweed mitogen as the stimulus, transformation of normal lymphocytes in sera of alcoholics with either normal or fatty livers was not significantly different from that obtained in pooled human serum (PHS). However, in sera of patients with either alcoholic hepatitis or inactive cirrhosis mean transformation was significantly reduced $(\mathrm{P}<0.001,<0.02$ respectively). With phytohaemagglutinin-P or concanavalin A as mitogens, suppression of transformation was not as marked but followed the same pattern. A significant negative correlation was observed between the magnitude of transformation and serum bilirubin and aspartate aminotransferase levels. An intra-patient comparison of the effects on transformation of normal lymphocytes by simultaneously collected peripheral and portal venous sera, and of peripheral sera obtained before and after portasystemic shunt surgery, indicated that the factor(s) responsible did not originate in the splanchnic circulation nor did it accumulate in the serum because of failed hepatic clearance. By performing transformation experiments in the presence of inhibitory patient sera diluted with PHS it was possible to show that these sera caused true inhibition of transformation rather than suppression due to failure to sustain cell culture because of nititional deficiencies. Inhibitory sera did not contain high levels of the enzyme thymidine phosphorylase and did not significantly inhibit binding of ${ }^{125}$ I-labelled mitogens to the lymphocyte surface. These findings indicate that the inhibitory effect of sera from alcoholics is of potential in vivo importance, that the effect increases with the degree of heptocyte damage, and that it is unrelated to the nonhepatic metabolic affects of chronic alcoholism.

Although it has been demonstrated that the sera of patients with a variety of hepatic disorders including viral hepatitis, primary biliary cirrhosis, chronic active hepatitis, and alcoholic liver disease can suppress in vitro lymphocyte transformation (Mella and Taswell, 1970; Hsu and Leevy, 1971; Fox et al., 1973; Newberry et al., 1973; Macsween and Thomas, 1973; Wands et al., 1975; Nakao et al., 1975; Brattig and Berg, 1976), the nature of this phenomenon is poorly understood in the majority

\footnotetext{
${ }^{1}$ This study was supported by the Alfred Hospital Henry Laurie Scholarship Fund.

${ }^{2} \mathrm{G}$. P. Young was supported in part by a Royal Australasian College of Physicians research grant. Present address: Division of Gastroenterology, Washington University School of Medicine, 660 S. Euclid Avenue, St. Louis, MO 63110, USA.

${ }^{3}$ Address for reprint requests: Dr F. J. Dudley, Gastroenterology Diagnostic Service, Alfred Hospital, Commercial Road, Prahran, Victoria 3181, Australia.

Received for publication 6 June 1979.
}

of instances. In chronic alcoholic patients it is not clear whether this effect is a consequence of liver damage or of the accompanying metabolic effects of alcoholism, including nutritional deficiency. This inhibitory effect of serum might also be an artefact in that it may be peculiar to the conditions of culture and hence of no possible relevance to in vivo lymphocyte function. Furthermore, existing reports of this phenomenon in alcoholic liver disease are conflicting and do not relate it to the spectrum of alcoholic liver damage (Hsu and Leevy, 1971; Newberry et al., 1973; Thestrup-Pedersen et al., 1976).

In an attempt to define the incidence and characteristics of this immunosuppressive phenomenon we have analysed the effect of sera from chronic alcoholics, with or without liver disease, on mitogeninduced transformation of normal lymphocytes. In addition, we attempted to identify the source of the factor(s) responsible for this suppression and ex- 
amined the possibility that this effect might be an in vitro artefact of no in vivo significance.

\section{Methods}

PATIENTS

Hospitalised patients suffering from chronic alcoholism, defined as a daily consumption of more than $100 \mathrm{~g}$ of alcohol for 10 years, were considered for admission to the study. Patients suffering from uraemia, pregnancy, uncorrected nutritional deficiency, suspected or confirmed autoimmune or viral liver disease, severe infection, neoplasia, or those receiving immunosuppressive therapy were excluded. Of those included, 39 were hospitalised because of suspected liver disease and the remainder for non-hepatic complications of chronic alcoholism. On the basis of clinical and biochemical findings and liver histology these patients have been arbitrarily divided into four groups

1 Alcoholics: normal liver function with normal histology or fatty change only on liver biopsy (10 patients).

2 Alcoholic hepatitis: defined as patients with histological or biochemical evidence of alcoholic hepatitis (Sherlock, 1975), with or without cirrhosis (14 patients)

3 Alcoholic cirrhosis: defined as patients with features of alcoholic cirrhosis (Sherlock, 1975) without evidence of hepatitis (15 patients).

4 Alcoholic cirrhosis and a surgical portasystemic shunt (12 patients).

Serum was obtained from each patient by venepuncture and then stored under sterile conditions at $-24^{\circ} \mathrm{C}$. Normal human pooled serum (PHS) was obtained from 100 normal blood donors and stored under identical conditions. Peripheral and splanchnic venous sera were simultaneously obtained from four patients with alcoholic cirrhosis undergoing portasystemic shunt surgery and from four non-alcoholic patients with normal liver function undergoing cholecystectomy. Approval to carry out this study was obtained from the Alfred Hospital Research Advisory Committee.

\section{LYMPHOCYTES}

Citrated peripheral venous blood samples, obtained from 18 healthy medical students and laboratory personnel, were centrifuged at $170 \mathrm{~g}$ for 20 minutes and the platelet rich plasma discarded. The buffy coat was suspended in Hanks' balanced salt solution (HBSS) and the mononuclear cells isolated by FicollHypaque gradient centrifugation (Böyum, 1968). After centrifugation at $800 \mathrm{~g}$ for 20 minutes the lymphocyte rich interface was removed and washed twice with HBSS. The cell pellet was suspended in Eagle's minimum essential medium (MEM), incubated at $37^{\circ} \mathrm{C}$ for one hour in a $5 \% \mathrm{CO}_{2} /$ air mixture and washed once with MEM. The final cell preparation had a platelet to mononuclear ratio of approximately $4: 1$ with a cell viability, as determined by trypan blue exclusion, of greater than $98 \%$. On stained smears of the lymphocyte preparations more than $90 \%$ of the cells were lymphocytes. For mitogen binding studies the mononuclear preparations obtained in this way were centrifuged through a discontinuous sucrose gradient (Perper et al., 1968) reducing the platelet to mononuclear ratio to $1: 4$.

\section{E-ROSETTES}

E-rosettes were measured in the presence of $20 \%$ PHS or patient serum according to Chisari and Edgington (1975). The lymphocyte:sheep erythrocyte ratio was $1: 40$.

\section{LYMPHOCYTE STIMULATION}

Lymphocyte stimulation was performed in microtitre plates containing $1.25 \times 10^{5}$ normal donor cells per well in $0.26 \mathrm{ml}$ of MEM containing penicillin and streptomycin, 100 units $/ \mathrm{ml}$, and supplemented with $20 \%$ patient or PHS. All tests were performed in triplicate and the mitogens used were purified phytohaemagglutinin (PHA-P; CSL, Melbourne), pokeweed mitogen (PWM; Grand Island Biological Co., New York), and concanavalin A (Con A; Calbiochem, Sydney). The concentrations of PHA-P used were $11 \cdot 5,46$, and $185 \mu \mathrm{g} / \mathrm{ml}$; PWM, 1, 4, and $10 \mu \mathrm{g} / \mathrm{ml}$; and Con A $10 \mu \mathrm{g} / \mathrm{ml}$. The cultures were incubated at $37^{\circ}$ for 72 hours in a humidified $5 \%$ $\mathrm{CO}_{2} /$ air atmosphere. Four hours before the end of the culture period, $0.5 \mu \mathrm{Ci}$ of (methyl- ${ }^{3} \mathrm{H}$ )-thymidine (The Radiochemical Centre, Amersham, $5 \mathrm{Ci} / \mathrm{mmol}$ was added to each well in $0.01 \mathrm{ml}$ of the culture medium. Trichloroacetic acid (TCA) precipitable material was collected on glass fibre filters by harvesting the cells with the aid of a multiple automated sample harvester (Titertek, Skatron, Norway) and radioactivity was determined in a Packard liquid scintillation counter. Results were expressed as mean $\mathrm{cpm}$ of triplicate cultures. The results obtained in $20 \%$ PHS for each batch of lymphocytes acted as the control. Patient groups were compared with controls using Student's $t$ test (paired).

\section{LYMPHOCYTE-MITOGEN BINDING}

Con $A$ and PWM were iodinated with ${ }^{125} I\left(\mathrm{Na}^{125} I\right.$, Australian Radiation Laboratory) using the lactoperoxidase method (Phillips and Morrison, 1970). Both compounds retained their mitogenic properties after this procedure. The labelled mitogens, at concentrations used in the transformation experi- 
ments, were added to lymphocytes suspended in MEM and $20 \%$ human serum. After incubation at room temperature for 15 minutes the cells were centrifuged at $1000 \mathrm{~g}$ for two minutes and washed twice with phosphate buffered saline. All experiments were performed in triplicate, and in the case of ${ }^{125} \mathrm{I}$ Con A, with and without 0.2 M $\alpha$ methyl-D-glucopyranoside ( $\alpha$ MG). ${ }^{125} \mathrm{I}$ binding was determined in a Nuclear Chicago gamma spectrometer. Results were expressed as $\mathrm{cpm} / 4 \times 10^{5}$ cells, the binding of mitogen to the plastic incubation tubes being subtracted in all cases. In the case of ${ }^{125} \mathrm{I}-\mathrm{Con} \mathrm{A}, \alpha$ MG inhibited binding to cells by up to $90 \%$ and the effect of serum on this specific binding only was considered.

\section{THYMIDINE PHOSPHORYLASE ASSAY}

These were carried out under the conditions applied in lymphocyte culture using a modified microassay technique (Pauly et al., 1977) in which the chromatography papers were developed by ascending chromatography. Conversion of tritiated thymidine
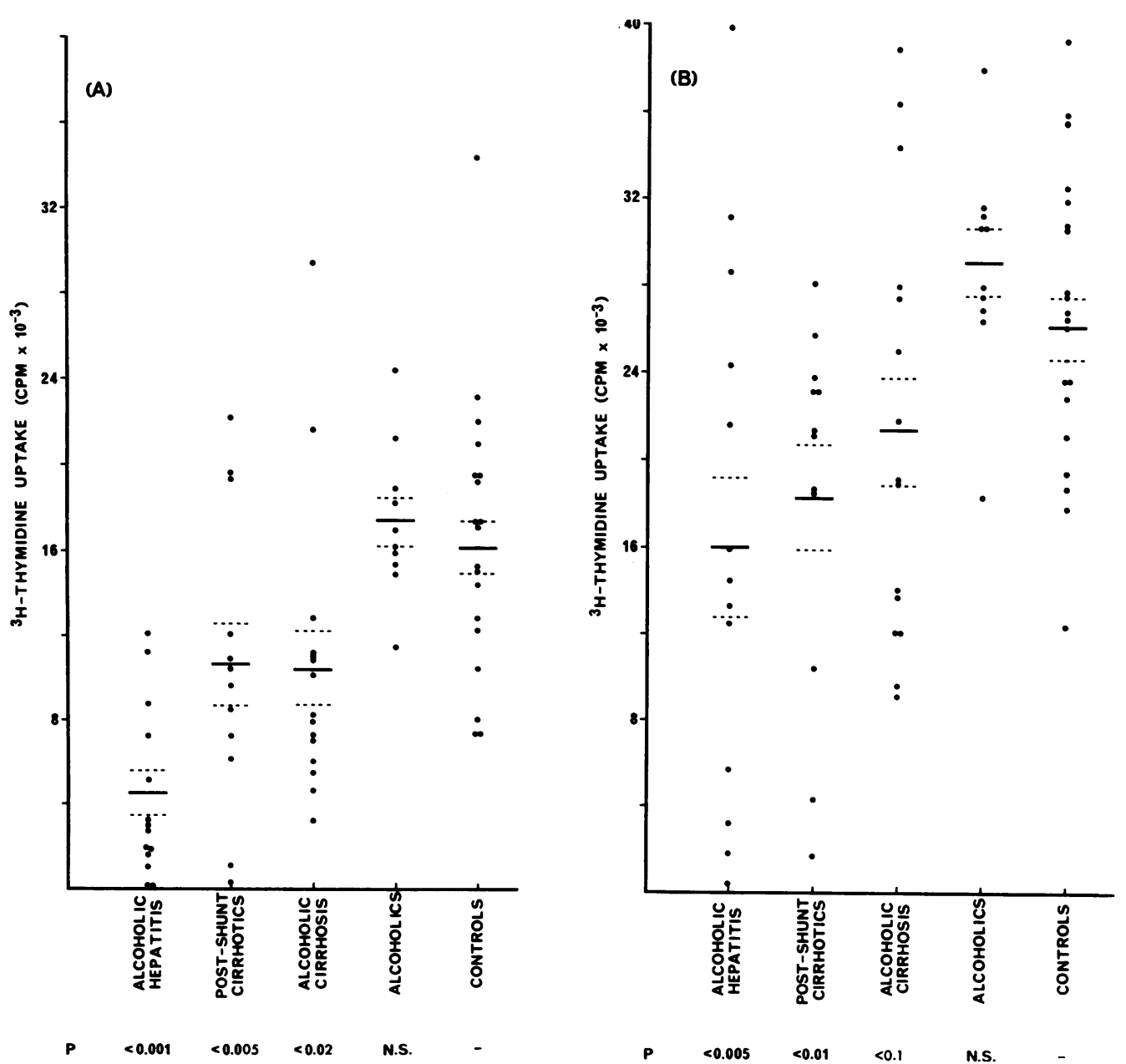

Fig. 1 Transformation of normal lymphocytes measured by ${ }^{3} \mathrm{H}$-thymidine uptake by trichloroacetic acid precipitates in individual test sera using optimal dosage of $(A)$ pokeweed mitogen and $(B)$ phytohaemagglutinin-P. Solid horizontal bars represent the mean for each group and the dotted lines the standard error of the mean. $P$ values refer to comparison between alcoholic sera sub-groups and pooled human serum ('controls') (see text). 'Alcoholics' refers to chronic alcoholics with normal or fatty livers. 
to tritiated thymine was determined by measuring radioactivity in the appropriate areas by liquid scintillation counting. Enzyme activity was expressed as the percentage tritiated thymidine degraded to tritiated thymine.

\section{Results}

The effects of sera from patients suffering from chronic alcoholism on PHA - or PWM - induced transformation of normal peripheral blood lymphocytes are shown in Fig. 1. With optimal concentration of PWM (Fig. 1a), transformation of normal lymphocytes in sera from patients with alcoholic hepatitis $\left(4526 \pm 1051 \mathrm{cpm} / 1.25 \times 10^{5}\right.$ lymphocytes, mean $\pm \mathrm{SE}, \mathrm{P}<0.001)$, alcoholic cirrhosis $(10457 \pm$ $1772 \mathrm{cpm}, \mathrm{P}<0.05$ ), and post-shunt alcoholic cirrhosis $(10595 \pm 2004 \mathrm{cpm}, P<0.05)$ was significantly reduced compared with normal controls $(16469 \pm 1508 \mathrm{cpm})$. In contrast, sera from alcoholics with normal liver function did not suppress transformation $(17337 \pm 1134 \mathrm{cpm})$. Using PHA-P suppression was less marked than with PWM (Fig. 1b). In non-shunted cirrhotics suppression with PHA-P was significant when compared with alcoholics with normal liver function $(P<0.05)$ but not when compared with control PHS $(0 \cdot 10>\mathrm{P}>0.05)$. In data not shown here, the same pattern of response was obtained using optimal concentration of Con A. Unstimulated cultures in PHS gave $134 \pm 75 \mathrm{cpm}$ $( \pm \mathrm{SE})$ and did not differ significantly from results in patient sera.

The observed inhibitory effect of sera on transformation was independent of the duration of culture, for similar findings were obtained in experiments in which cells were harvested three, four, and five days after mitogen stimulation. Supraoptimal concentrations of PHA-P or PWM failed to overcome observed suppression in the large majority of patients (Fig. 2). Transformation in sera from the group of cirrhotic patients with surgical portasystemic shunts did show significant improvement with increasing doses of PHA-P, although this did not reach a significantly higher level than transformation with supraoptimal doses in sera from cirrhotics without surgical shunts.

A significant degree of negative correlation was observed between the magnitude of transformation using either mitogen and the serum bilirubin level $(r=-0.5138, \mathrm{P}<0.001$ for PHA-P; $r=-0.4909$, $\mathrm{P}<0.01$ for PWM), between serum aspartate aminotransferase levels and PWM-induced transformation $(r=-0.3301, \quad \mathrm{P}<0.01)$, and between $\quad x$-globulin levels and PHA-P-induced transformation $(r=$ $-0.3650, \quad P<0.05)$. No significant correlation (positive or negative) could be demonstrated

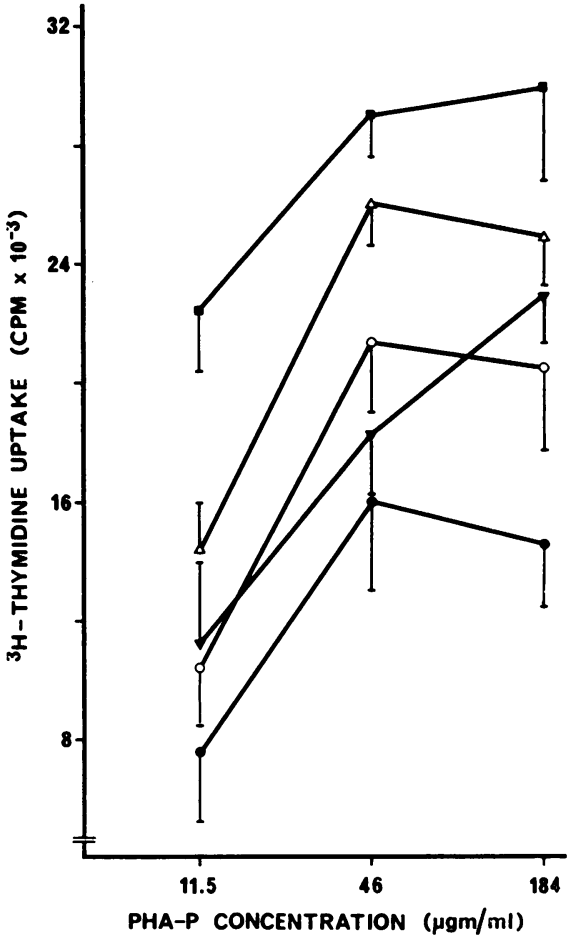

Fig. 2 Mean phytohaemagglutinin (PHA-P) stimulated transformation of normal lymphocytes in sera from each subgroup of chronic alcoholics and in pooled human serum (PHS). Vertical bars represent standard error of the mean. Alcoholic hepatitis $\bigcirc$; alcoholic cirrhosis $\bigcirc$; post-shunt cirrhotics $\nabla ; P H S \triangle$; alcoholics without liver disease $\mathbf{}$.

between the magnitude of transformation and serum levels of albumin, alkaline phosphatase, suppression of E-rosette formation, fibrin degradation products, and very low density lipoproteins. No patient was $\alpha$-foetoprotein positive.

\section{EFFECT OF PORTACAVAL SHUNTING ON} SERUM INHIBITION

Peripheral blood sera obtained from nine patients with cirrhosis and portal hypertension just before and one month after portasystemic shunt surgery were studied. With optimal concentration of PHA-P, transformation of normal lymphocytes (mean $\pm S E$ ) using preshunt sera was $19102 \pm 3039 \mathrm{cpm}$ and with postshunt sera was $21435 \pm 3848 \mathrm{cpm}(\mathrm{P}>0 \cdot 2)$. Results obtained with other concentrations of PHA-P, and with PWM as the mitogen, confirmed that the suppressing effect of these sera was not accentuated by an increase in degree of portasystemic shunting. All patients appeared to have patent shunts at the time of study on the basis of 
clinical findings and scintisplenoportography in some patients.

In either controls or alcoholic cirrhosis patients, comparison of the effects of corresponding portal versus peripheral sera on transformation revealed no significant differences.

\section{MECHANISM AND NATURE OF INHIBITION BY PATIENT SERA}

Thymidine phosphorylase assays

Thymidine phosphorylase activity was measured in PHS and 11 suppressing sera. Mean enzyme activity $( \pm$ SE) in $20 \%$ PHS was $14.0 \pm 1.0 \%$. The mean level in $20 \%$ patient sera was $9 \cdot 0 \pm 1 \cdot 1 \%$ (range $1 \cdot 4-27 \cdot 8 \%$ ) with only one patient having a mean value in excess of that for PHS.

\section{Lymphocyte-mitogen binding}

Eleven patient sera most suppressive for Con Ainduced transformation (mean suppression of $40.4 \%$ ) failed to cause a significant reduction of specific Con A binding - mean binding in control PHS was $5713 \pm 429 \mathrm{cpm} / 4 \times 10^{5}$ cells $( \pm \mathrm{SE})$ against $5369 \pm$ 428 in patient sera. Similarly, eight sera suppressive for PWM (mean suppression of $57.2 \%$ ) gave a mean binding of $2095 \pm 180 \mathrm{cpm} / 4 \times 10^{5}$ cells $( \pm \mathrm{SE})$ against $2597 \pm 407$ in PHS. These differences are not significant.

\section{Effect of dilution and dialysis}

To ascertain if the inhibitory effect of patient sera was principally a consequence of a nutrient deficiency, transformation experiments were simultaneously performed in two sets of culture conditions which were then compared: (1) PHS only in the cultures, in proportions of $5 \%, 10 \%, 15 \%$, and $20 \%$; (2) PHS mixed with individual patient sera in ratios of $3: 1,2: 2,1: 3$, and $0: 4$ respectively, the total serum concentration being maintained at $20 \%$. The results are shown in Fig. 3. Using sera from five patients, with PWM as the mitogenic stimulus, the addition of $10 \%$ or $5 \%$ patient serum to $10 \%$ or $15 \%$ PHS respectively, reduced transformation. Seven patients were similarly tested with PHA-P and five with Con-A and similar patterns were observed.

Dialysis of sera against culture media did not reduce their inhibitory effect and heating to $56^{\circ} \mathrm{C}$ for one hour did not significantly alter the degree of inhibition of transformation.

\section{Discussion}

Previous reports of the effect of sera from patients with alcoholic liver disease on lymphocyte transformation are conflicting. Whereas Hsu and Leevy (1971) and Newberry et al. (1973) found that sera from patients with alcoholic cirrhosis suppressed in vitro lymphocyte transformation, a more recent study using heat-treated sera (Thestrup-Pedersen $e t$ al., 1976) failed to find such an effect. In the present report it has been demonstrated that the sera of patients with chronic alcoholism have wide-ranging effects on lymphocyte transformation, possibly explaining these conflicting reports. In addition, we found significant inhibition to occur only in sera from those with cirrhosis or alcoholic hepatitis, suggesting that alcoholic liver damage is responsible rather than nonhepatic metabolic effects of alcoholism. The slight possibility that suppression resulted from failure to correct subtle nutritional deficiencies despite clinical and biochemical normality seems unlikely, as the results of serum dilution experiments (Fig. 3) can be explained only by the presence of an active inhibitor(s) of tritiatedthymidine uptake.

The mechanism by which these sera cause suppression remains obscure but a number of alternatives peculiar to in vitro conditions, and thus of no in vivo consequence, have been excluded. The enzyme thymidine phosphorylase has been shown to be present in plasma and has prompted the suggestion that alteration in its activity may lead to erroneous interpretation of these effects of differing sera on lymphocyte transformation (Pauly et al., 1977). However, assay of this enzyme in control and

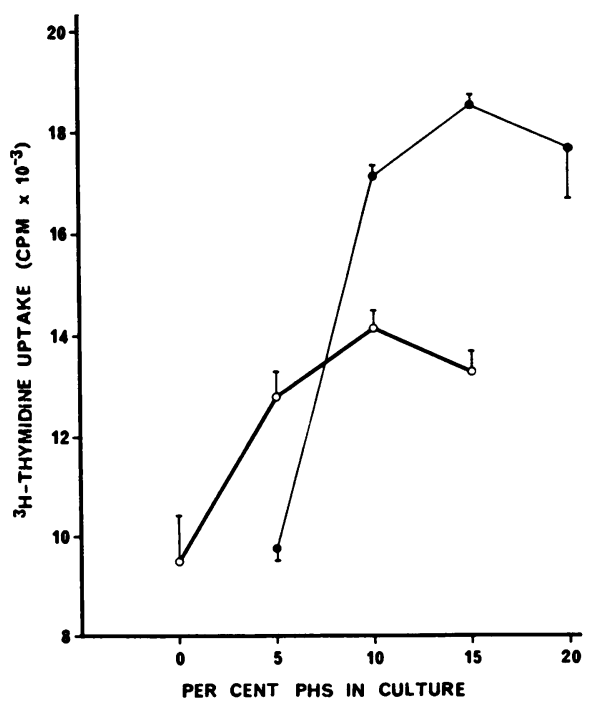

Fig. 3 The effect on pokeweed mitogen-induced lymphocyte transformation of varying percentages of pooled human serum (PHS) only (O-O) or mixing with patient serum $(\bigcirc-\bigcirc)$ in varying proportions to keep total serum concentration at $20 \%$. The patient sera curve $(\bigcirc-\bigcirc)$ represents the mean for five different sera. Vertical bars represent $S E$ of the mean. 
inhibitory sera demonstrated that changes in its activity could not account for the observed suppression. It is also possible that sera may interfere with mitogen-lymphocyte interaction by causing steric hindrance with surface receptors for mitogen (Boldt et al., 1972; Nicolson, 1974) or by serum protein binding of large amounts of the mitogenic stimulant (Humphrey et al., 1974). These effects appear unlikely in this study, however, as ${ }^{125}$ I-PWM and ${ }^{125} \mathrm{I}$-Con $\mathrm{A}$ binding experiments showed that quantitative mitogen binding to lymphocytes was not significantly reduced by inhibitory sera; many of the suppressing sera suppressed transformation to all three mitogens, each having different receptor specificity (Nicolson, 1974); and with only occasional exceptions could inhibition be overcome by increasing the dose of mitogen.

As inhibition is most marked in sera from patients with alcoholic hepatitis, and as it correlates with bilirubin and AST levels, hepatocyte damage appears important in the development of inhibitory factors in the serum (SIF). It is not clear whether SIF is released from damaged hepatocytes or not. The effects of peripheral and portal venous sera on transformation were similar for both patients and non-alcoholic controls, and, as SIF did not increase after portasystemic shunt surgery, it is unlikely that SIF originates in the splanchic circulation and gains access to the peripheral circulation either because of failed clearance by the damaged hepatocyte or because of portasystemic shunting.

In hepatic cirrhosis failure of hepatic clearance of gut antigens results in an increased antigenic challenge, which appears to be a major factor leading to the hypergammaglobulinaemia seen in many patients (Zinneman, 1975). In vitro animal studies have shown that sensitised lymphocytes exposed simultaneously to multiple antigens, produce soluble factors inhibitory to antibody production (Kempf and Rubin, 1977) and lymphocyte proliferation (Thomas et al., 1975) SIF may thus be a non-specific immunoregulatory factor released by lymphoid cells of patients with alcoholic liver disease as a consequence of hyperstimulation of the immune system.

These observations, that suppression of lymphocyte transformation does not appear to result from (1) nutrient deficiencies in the sera, (2) increased serum thymidine phosphorylase levels, or (3) interference with lymphocyte-mitogen interaction, are of some importance. They suggest the possibility that SIF acts at a point common to both lectin- and antigen-induced blastogenesis and thus could modify in vivo function of lymphocytes with immunological consequences. Both the susceptibility of alcoholics, particularly with liver disease, to various infections
(Straus and Berenyi, 1971, 1973; Berenyi et al., 1974; Leevy et al., 1976; Isselbacher, 1977) and the impairment of delayed sensitivity reactions observed in alcoholics with liver disease (but not in those without liver disease) (Berenyi et al., 1974) may be in part a consequence of inhibition of in vivo lymphocyte function by SIF. This effect of SIF on nucleotide incorporation may inhibit lymphocyte turnover and explain the lymphopenia encountered in patients with alcoholic liver disease (Young et al., 1979).

The presence of SIF in the sera of subjects with alcoholic liver disease represents a further metabolic abnormality in these patients, which may have important immunological clinical consequences. Further characterisation of the nature of SIF, the mechanism by which it affects lymphocyte function, and its in vivo consequences seems warranted.

We wish to thank Mr Ian Rose for his excellent technical assistance and $\mathrm{Dr} \mathrm{A}$. Goh and Dr J. Rolland for their helpful advice.

\section{References}

Berenyi, M. R., Straus, B., and Cruz, D. (1974). In vitro and in vivo studies of cellular immunity in alcoholic cirrhosis. American Journal of Digestive Diseases, 19, 199-205.

Boldt, D., Skinner, A. M., and Kornfeld, S. (1972). Studies of two subpopulations of human lymphocytes differing in responsiveness to concanavalin A. Journal of Clinical Investigation, 51, 3225-3234.

Böyum, A. (1968). Isolation of mononuclear cells and granulocytes from human blood. Scandinavian Journal of Clinical and Laboratory Investigation, 21, suppl. 97, 77-89.

Brattig, N., and Berg, P. A. (1976). Serum inhibitory factors (SIF) in patients with acute and chronic hepatitis and their clinical significance. Clinical and Experimental Immunology, 25, 40-49.

Chisari, F. V., and Edgington, T. S. (1975). Lymphocyte E rosette inhibitory factor: a regulatory serum lipoprotein. Journal of Experimental Medicine, 142, 1092-1107.

Fox, R. A., Dudley, F. J., Samuels, M., Milligan, J., and Sherlock, S. (1973). Lymphocyte transformation in response to phytohaemagglutinin in primary biliary cirrhosis: the search for a plasma inhibitory factor. Gut, 14, 89-93.

Hsu, C. C. S., and Leevy, C. M. (1971). Inhibition of PHA-stimulated lymphocyte transformation by plasma from patients with advanced alcoholic cirrhosis. Clinical and Experimental Immunology, 8, 749-760.

Humphrey, G. B., Lankford, J., Oleinick, S., and Nitschke, R. (1974). Leukemic serum inhibition of phytohemagglutinin-induced transformation. Journal of Immunology, 113, 63-69.

Isselbacher, K. J. (1977). Metabolic and hepatic effects of alcohol. New England Journal of Medicine, 296, 612-616. 
Kempf, K. E., and Rubin, A. S. (1977). Transient suppression of the humoral immune response mediated by a factor derived from specifically activated, doubly primed lymphoid cells. Journal of Immunology, 119, 517-523.

Leevy, C. M., Chen, T., Luisada-Opper, A., Kanagasundarum, N., and Zetterman, R. (1976). Liver disease of the alcoholic: role of immunologic abnormalities in pathogenesis, recognition and treatment. Progress in Liver Diseases, 5, 516-530.

Macsween, R. N. M., and Thomas, M. A. (1973). Lymphocyte transformation by phytohaemagglutinin (PHA) and purified protein derivative (PPD) in primary biliary cirrhosis. Clinical and Experimental Immunology, 15, 523-533.

Mella, B. A., and Taswell, H. F. (1970). Suppression of leukocytic mitosis by sera of hepatitis-implicated donors. American Journal of Clinical Pathology, 53, 141-144.

Nakao, M., Mizoguchi, Y., Monna, T. et al. (1975). Studies on the sub-population and function of peripheral lymphocytes, and inhibitor to PHA stimulation existing in the serum of patients with liver disease. Gastroenterologica Japonica, 10, 307-315.

Newberry, W. M., Shorey, J. W., Sanford, J. P., and Combes, B. (1973). Depression of lymphocyte reactivity to phytohemagglutinin by serum from patients with liver disease. Cellular Immunology, 6, 87-97.

Nicolson, G. L. (1974). The interactions of lectins with animal cell surfaces. International Review of Cytology, 39, 89-190.

Pauly, J. L., Schuller, M. G., Zelcer, A. A., Kirss, T. A., Gore, S. S., and Germain M. J. (1977). Identification and comparative analysis of thymidine phosphorylase in the plasma of healthy subjects and cancer patients. Journal of the National Cancer Institute, 58, 1587-1590.

Perper, R. J., Zee, T. W., and Mickelson, M. M. (1968). Purification of lymphocytes and platelets by gradient centrifugation. Journal of Laboratory and Clinical Medicine, 72, 842-848.

Phillips, D. R., and Morrison, M. (1970). The arrangement of proteins in the human erythrocyte membrane. Biochemical and Biophysical Research Communications, 40, 284-289.

Sherlock, S. (1975). Diseases of the Liver and Biliary System, 5th edn., Blackwell: Oxford.

Straus, B., and Berenyi, M. R. (1973). Infection and immunity in alcholic cirrhosis. Mt Sinai Journal of Medicine NY, 40, 631-640.

Straus, B., Berenyi, M. R., Huang, J. M., and Straus, E. (1971). Delayed hypersensitivity in alcoholic cirrhosis. American Journal of Digestive Diseases, 16, 509-516.

Thestrup-Pedersen, K., Ladefoged, K., and Anderson, P. (1976). Lymphocyte transformation test with liverspecific protein and phytohaemagglutinin in patients with liver disease. Clinical and Experimental Immunology, 24, 1-8.

Thomas, D. W., Roberts, W. K., and Talmage, D. W. (1975). Regulation of the immune response: production of a soluble suppressor by immune spleen cells in vitro. Journal of Immunology, 114, 1616-1622.

Wands, J. R., Perrotto, J. L., Alpert, E., and Isselbacher, K. J. (1975). Cell-mediated immunity in acute and chronic hepatitis. Journal of Clinical Investigation, 55, 921-929.

Young, G. P., Van der Weyden, M. B., Rose, I. S., and Dudley, F. J. (1979). Lymphopenia and lymphocyte transformation in alcoholics. Experientia, 35, 268-269.

Zinneman, H. H. (1975). Autoimmune phenomena in alcoholic cirrhosis. American Journal of Digestive Diseases, 20, 337-345. 